\title{
Epigenome-Wide Association Study of Prostate Cancer in African Americans Identifies DNA Methylation Biomarkers for Aggressive Disease
}

\author{
Yifan $\mathrm{Xu}^{1,+}{ }^{\dagger}$, Chia-Wen Tsai ${ }^{1,2,+}$, Wen-Shin Chang ${ }^{1,2,+}$, Yuyan Han ${ }^{3} \oplus$, Maosheng Huang ${ }^{1}$, Curtis A. Pettaway ${ }^{4}$, \\ Da-Tian Bau ${ }^{2,5, \ddagger(1)}$ and Jian Gu ${ }^{1, *, \ddagger}$
}

Citation: Xu, Y.; Tsai, C.-W.; Chang, W.-S.; Han, Y.; Huang, M.; Pettaway, C.A.; Bau, D.-T.; Gu, J.

Epigenome-Wide Association Study of Prostate Cancer in African

Americans Identifies DNA

Methylation Biomarkers for Aggressive Disease. Biomolecules 2021, 11, 1826. https://doi.org/10.3390/ biom 11121826

Academic Editors: Cecilia M. P. Rodrigues and Prakash Kulkarni

Received: 9 October 2021

Accepted: 1 December 2021

Published: 3 December 2021

Publisher's Note: MDPI stays neutral with regard to jurisdictional claims in published maps and institutional affiliations.

Copyright: (c) 2021 by the authors. Licensee MDPI, Basel, Switzerland. This article is an open access article distributed under the terms and conditions of the Creative Commons Attribution (CC BY) license (https:/ / creativecommons.org/licenses/by/ $4.0 /)$.
1 Department of Epidemiology, The University of Texas MD Anderson Cancer Center, Houston, TX 77030, USA yxu13@mdanderson.org (Y.X.); wenwen816@gmail.com (C.-W.T.); halittlemelon@hotmail.com (W.-S.C.); mshuang@mdanderson.org (M.H.)

2 Terry Fox Cancer Research Laboratory, China Medical University Hospital, Taichung 404332, Taiwan; artbau2@gmail.com

3 School of Biological Sciences, University of Northern Colorado, Greeley, CO 80639, USA; yuyan.han@unco.edu

4 Department of Urology, The University of Texas MD Anderson Cancer Center, Houston, TX 77030, USA; cpettawa@mdanderson.org

5 Department of Bioinformatics and Medical Engineering, Asia University, Taichung 41354, Taiwan

* Correspondence: jiangu@mdanderson.org; Tel.: +1-(713)-792-8016; Fax: +1-(713)-792-2145

+ Co-first author.

$\ddagger$ Co-last author.

Abstract: DNA methylation plays important roles in prostate cancer (PCa) development and progression. African American men have higher incidence and mortality rates of PCa than other racial groups in U.S. The goal of this study was to identify differentially methylated CpG sites and genes between clinically defined aggressive and nonaggressive PCa in African Americans. We performed genome-wide DNA methylation profiling in leukocyte DNA from 280 African American PCa patients using Illumina MethylationEPIC array that contains about $860 \mathrm{~K} \mathrm{CpG}$ sties. There was a slight increase of overall methylation level (mean $\beta$ value) with the increasing Gleason scores (GS $=6$, GS $=7$, $\mathrm{GS} \geq 8, \mathrm{P}$ for trend $=0.002$ ). There were 78 differentially methylated $\mathrm{CpG}$ sites with $\mathrm{P}<10^{-4}$ and 9 sites with $\mathrm{P}<10^{-5}$ in the trend test. We also found 77 differentially methylated regions/genes (DMRs), including 10 homeobox genes and six zinc finger protein genes. A gene ontology (GO) molecular pathway enrichment analysis of these 77 DMRs found that the main enriched pathway was DNA-binding transcriptional factor activity. A few representative DMRs include HOXD8, SOX11, ZNF-471, and ZNF-577. Our study suggests that leukocyte DNA methylation may be valuable biomarkers for aggressive PCa and the identified differentially methylated genes provide biological insights into the modulation of immune response by aggressive PCa.

Keywords: prostate cancer; African American; aggressive disease; DNA methylation; leukocytes

\section{Introduction}

Prostate cancer (PCa) is the most common cancer and second leading cause of cancer death in American men, with an estimated 248,530 new cases and 34,130 deaths from PCa in the U.S. in 2021 [1]. African American men have the highest incidence and mortality rates among all the racial/ethnic groups in the U.S. [2,3]. Prostate-specific antigen (PSA) testing has enabled the detection of PCa at early stages and greatly improved the survival of PCa. The majority of PSA screening-detected PCa are localized, indolent, and not life-threatening. However, most of the PCa patients opt to receive upfront aggressive therapies (radical proctectomy and radiotherapy) that are often associated with significant morbidity, thus overdiagnosis and overtreatment for localized PCa patients have become 
a major clinical problem. The major reason for the overtreatment is that is the current prognostic algorithms are predominantly composed of clinical variables, such as PSA at diagnosis, Gleason Score (GS), and tumor stage, which are not accurate enough to distinguish aggressive from nonaggressive diseases at the time of disease presentation. Patients with the same clinical features, for example, those with the same GS of 7, often have completely different prognoses-some are dormant and some progress to metastatic disease. Independent biomarkers may be able to supplement clinical variables to predict the clinical course of PCa patients and improve risk stratification of PCa patients for better-informed clinical management.

PCa tumors, particularly localized tumors, have fewer genetic mutations than other adult solid tumors $[4,5]$. In a large-scale whole genome and whole exome sequence analysis of nearly 500 localized PCa tumors (GS $\leq 7$ ), no gene was mutated in more than $10 \%$ of tumors, and only six genes were mutated in more than $2 \%$ of tumors [6]. The mutation frequencies in African American PCa patients are the lowest among all the different racial groups [7]. These somatic mutations are not associated with PCa aggressiveness. Epigenetic changes, including DNA methylation, on the other hand, play prominent roles in PCa development and progression, and may serve as promising biomarkers for $\mathrm{PCa}$ diagnosis and prognosis [8,9]. A commercially available test, ConfirmMDx, which measures CpG island methylation of GSTP1, RASSF1, and APC in histopathologically negative prostate core biopsies, has been used clinically to predict $\mathrm{PCa}$ and high-grade $\mathrm{PCa}$ in repeat biopsies [10-12]. In addition to DNA methylation in tumor tissues, DNA methylation in peripheral blood leukocytes has also attracted great interest as a predictor of cancer risks and outcomes [13-23]. Recent studies have applied an epigenome-wide association study (EWAS) approach using Illumina's high-density methylation arrays to identify specific CPG sites in leukocyte DNA that are differentially methylated between European ancestry PCa cases and controls, as well as between low-grade and high-grade PCa patients [21-23]. However, no study has performed whole epigenome-wide profiling of CpG site methylations in leukocytes of African American PCa patients.

In this study, we performed an EWAS of African American PCa patients and identified specific leukocyte $\mathrm{CpG}$ site methylations that may serve as biomarkers for the aggressive $\mathrm{PCa}$ in African Americans.

\section{Materials and Methods}

\subsection{Study Population}

This study included 280 self-reported African American men with histologically confirmed prostate adenocarcinoma. All patients were treated and followed up at the University of Texas MD Anderson Cancer Center. Blood specimens were collected at the time of diagnosis, before treatments. Clinical and follow-up data, which included date of diagnosis, performance status, clinical stage, Gleason scores, PSA levels at diagnosis and follow-up, and treatment (e.g., prostatectomy, radiotherapy, and hormone therapy), were extracted from electronic medical records. The study was approved by the Institutional Review Board of MD Anderson Cancer Center. All patients signed an informed consent form.

\subsection{Whole Genome Methylation Profiling Using Illumina Human MethylationEPIC Beadchip}

The whole genome CPG site methylation profiling was performed using Illumina human MethylationEPIC Beadchip, as previously described [22,23]. The MethylationEPIC Beadchip contains over 850,000 CpG sites across the human genome, among which 54\% are located within gene promoters, $30 \%$ in gene bodies, and $16 \%$ in intergenic regions $(16 \%)$ [24]. The CpG sites in CPG island are enriched on the MethylationEPIC chip, accounting for $19 \%$ of all the CpG sites on the chip [24]. Briefly, $500 \mathrm{ng}$ genomic DNA from peripheral blood was treated with sodium bisulfite using the EZ DNA Methylation-Gold Kit (Zymo Research, Irvine, CA) following manufacturer's protocol. Bisulfite-treated DNA was hybridized to the MethylationEPIC chip according to the manufacturer's protocol. 
Beadchips were scanned on an Illunima HiScan SQ. The fluorescence intensities of the images were extracted using Genome Studio Methylation Module.

\subsection{Bioinformatics and Data Analyses}

Raw fluorescence intensity data (idat files) were processed and normalized using the minfi R package [25]. The methylation status of each $\mathrm{CpG}$ site was shown as $\beta$ value, calculated as the ratio of the fluorescence intensity signals of the methylated (M) and unmethylated $(\mathrm{U})$ alleles [25]. $\beta$ values range between 0 (non-methylated) and 1 (completely methylated). The batch effect was removed using the ComBat function from $\mathrm{R}$ package [26]. The variation in peripheral blood white cell proportions was controlled for using cell proportion estimates generated by the estimateCellCounts function in minfi $\mathrm{R}$ package [27]. The differentially methylated $\mathrm{CpG}$ sites (DMCs) were identified by comparing the $\beta$ values of each CpG site between GS $=6, G S=7$, and GS $\geq 8$ groups using a trend test. To determine the prognostic value of each DMC, we dichotomized patients into highand low-methylation groups based on the median $\beta$ value and used the low-methylation group as the reference group. A multivariable Cox proportional hazards model was used to estimate the hazard ratio (HR) and $95 \%$ confidence interval (CI) for the associations of each DMC and biochemical recurrence (BCR), adjusting for age, PSA level, Gleason score, clinical stage, and treatment. We also identified differentially methylated regions/genes (DMRs). We set the criteria of DMR as having at least 7 consecutive CpG sites and the largest distance between each CpG site as 500 base pairs.

\section{Results}

\subsection{Patient Characteristics}

We performed methylation profiling in leukocyte DNA from 280 African American PCa patients. Table 1 shows the selected patient characteristics. The median age of patients was 58 (range, 39-83) years. The majority (57.9\%) were never smokers, whereas $12.1 \%$ were current smokers and $30 \%$ former smokers. Nearly half (49.1\%) of patients were obese (body mass index $[\mathrm{BMI}] \geq 30)$ and another $36.8 \%$ were overweight $(25 \leq \mathrm{BMI}<30)$. There were $82(29.3 \%) \mathrm{GS}=6,162(58.8 \%) \mathrm{GS}=7$, and $36(12.9 \%) \mathrm{GS} \geq 8$ patients. The patients were predominantly stage I (57.9\%) and stage II (36.1\%) patients and had PSA $<10 \mathrm{ng} / \mathrm{mL}(77.2 \%)$.

Table 1. Selected patient characteristics.

\begin{tabular}{cc}
\hline Variables & $\mathbf{N ~ ( \% )}$ \\
\hline Age, median (range) & $58(39-83)$ \\
Smoking status & \\
Never smoker & $162(57.9)$ \\
Former smoker & $84(30.0)$ \\
Current smoker & $34(12.1)$ \\
Body mass index (BMI) & \\
$<25$ & $33(14.1)$ \\
$25 \leq$ BMI <30 & $86(36.8)$ \\
$\geq 30$ & $115(49.1)$ \\
Gleason Score & \\
6 & $82(29.3)$ \\
7 & $162(58.8)$ \\
$\geq 8$ & $36(12.9)$ \\
Stage & $162(57.9)$ \\
T1 & $101(36.1)$ \\
T2 & $11(3.9)$ \\
T3 & $6(2.1)$ \\
PSA at diagnosis & \\
$<10$ ng/mL & $216(77.2)$ \\
$\leq 10$ and $<20 \mathrm{ng} / \mathrm{mL}$ & $39(13.9)$ \\
$\geq 20 \mathrm{ng} / \mathrm{mL}$ & $25(8.9)$ \\
\hline
\end{tabular}




\subsection{Leukocyte CpG Methylation Pattern in African American PCa Patients}

We first compared the genome-wide $\mathrm{CpG}$ methylation levels between patients with different Gleason scores. Overall, the mean methylation levels of all the assayed $860 \mathrm{~K} \mathrm{CpG}$ sites were slightly higher in GS $\geq 8$ (mean $\beta=0.5091$ ) and GS $=7$ (mean $\beta=0.5088$ ) than in $\mathrm{GS}=6$ (mean $\beta=0.5076$ ) patients (P for trend $=0.002$ ). As expected, the mean methylation levels were dramatically different depending on the locations of $\mathrm{CpG}$ sites, with $\mathrm{CpG}$ sites within or near the transcriptionally active regions showing the lowest methylation levels (Figure 1). CpG islands play critical roles in regulating gene expression and mostly have very low methylation (mean $\beta=0.189$ ), allowing active transcription, while CpG sites outside of $C p G$ islands have much higher methylations (mean $\beta=0.420,0.645$, and 0.625 for CpG sites in shores, shelfs, and open seas relative to islands). When CpG sites were grouped by locations relative to genes, the mean $\beta$ values showed a progressive increase associated with the increased distance to the core promoter. CpG sites within 200 base pairs of the transcription start site (TSS200) had the lowest methylation (mean $\beta=0.187$ ), followed by those in the Exon 1 (mean $\beta=0.218$ ), TSS-1500 (within 1500 base pairs of TSS) (mean $\beta=0.378), 5^{\prime}$ untranslated region (5'-UTR) (mean $\beta=0.417$ ), intergenic region (IGR) (mean $\beta=0.566$ ), gene body (mean $\beta=0.608$ ), and $3^{\prime}$-UTR (mean $\beta=0.653$ ) (Figure 1 ).

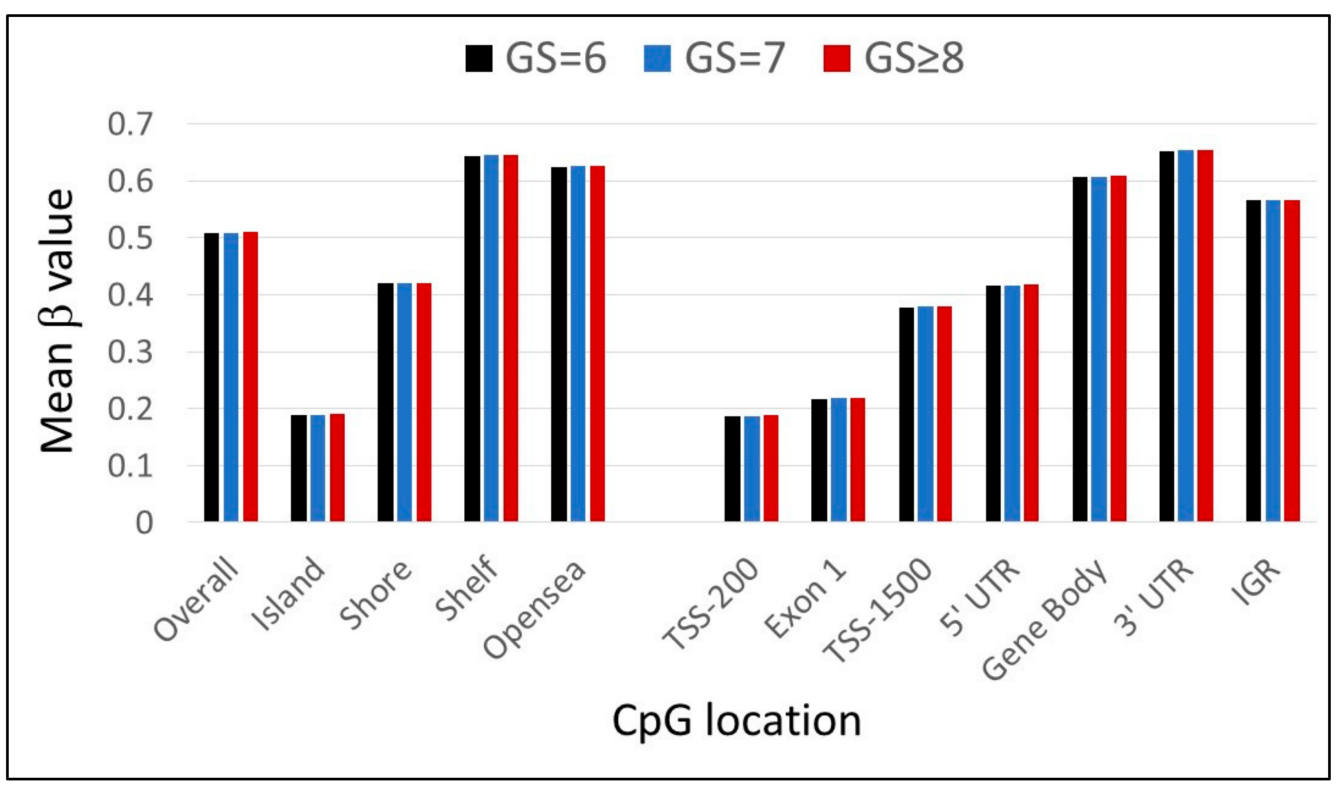

Figure 1. Comparisons of mean $\beta$ values of $C p G$ sites by locations of $C p G$ sites relative to islands and gene structure to show the overall leukocyte DNA methylation in African American prostate cancer patients with different Gleason scores. Abbreviations: TSS200: within 200 bp of the transcription start site (TSS); TSS1500: within 1500 bp of the TSS; UTR: untranslated region; IGR: intergenic regions.

We then performed a trend test to identify individual $\mathrm{CpG}$ sites that showed significant trends of increasing or decreasing methylation associated with increasing Gleason scores. There were 52,456 differentially methylated $\mathrm{CpG}$ sites with a nominal significance $(\mathrm{P}<0.05)$, 10,734 CpG sites with $\mathrm{P}<0.01,993$ sites with $\mathrm{P}<0.001,78$ sites with $\mathrm{P}<10^{-4}$, and 9 sites with $\mathrm{P}<10^{-5}$. About $80 \%$ of these DMCs showed a progressive increase of methylation with increasing GS (Table 2). 
Table 2. Top Differentially methylated CpG sites $\left(\mathrm{P}<1 \times 10^{-4}\right)$ between different Gleason scores.

\begin{tabular}{|c|c|c|c|c|c|c|c|c|c|}
\hline \multirow[b]{2}{*}{ CpG ID } & \multicolumn{3}{|c|}{$\beta$ Value } & \multirow[b]{2}{*}{$\begin{array}{l}\text { P for } \\
\text { Trend }\end{array}$} & \multirow[b]{2}{*}{ Chr. } & \multirow[b]{2}{*}{ Gene } & \multirow[b]{2}{*}{ CpG Location } & \multicolumn{2}{|c|}{ Cox Analysis for BCR } \\
\hline & $\mathrm{GS}=6$ & $\mathrm{GS}=7$ & $\mathrm{GS} \geq 8$ & & & & & HR $(95 \%$ CI $)$ & $\begin{array}{c}p \\
\text { Value }\end{array}$ \\
\hline $\operatorname{cg} 11039604$ & 0.926 & 0.94 & 0.952 & $1.17 \times 10^{-6}$ & 3 & $\mathrm{~N} / \mathrm{A}$ & IGR-Open sea & $1.16(0.43-3.09)$ & 0.768 \\
\hline cg15610955 & 0.843 & 0.85 & 0.865 & $3.32 \times 10^{-6}$ & 2 & GPR39 & Body-Open sea & $1.1(0.43-2.8)$ & 0.834 \\
\hline cg01097384 & 0.313 & 0.339 & 0.395 & $3.42 \times 10^{-6}$ & 11 & $\mathrm{~N} / \mathrm{A}$ & IGR-Island & $1.14(0.45-2.91)$ & 0.782 \\
\hline cg02919721 & 0.674 & 0.692 & 0.712 & $3.84 \times 10^{-6}$ & 1 & $\mathrm{~N} / \mathrm{A}$ & Open sea & $1.51(0.59-3.84)$ & 0.387 \\
\hline cg10283070 & 0.803 & 0.801 & 0.773 & $4.46 \times 10^{-6}$ & 9 & OR1B1 & TSS1500-Open sea & $0.74(0.31-1.75)$ & 0.488 \\
\hline cg22367191 & 0.259 & 0.284 & 0.3 & $5.73 \times 10^{-6}$ & 6 & $\mathrm{~N} / \mathrm{A}$ & IGR-Shore & $0.74(0.28-1.91)$ & 0.529 \\
\hline cg18092924 & 0.809 & 0.82 & 0.833 & $7.27 \times 10^{-6}$ & 20 & DIDO1 & 5'UTR-Shelf & $1.02(0.42-2.46)$ & 0.968 \\
\hline cg02073492 & 0.845 & 0.859 & 0.866 & $7.48 \times 10^{-6}$ & 6 & $\mathrm{~N} / \mathrm{A}$ & IGR-Open sea & $0.94(0.38-2.34)$ & 0.901 \\
\hline cg03890037 & 0.157 & 0.168 & 0.197 & $7.50 \times 10^{-6}$ & 5 & LVRN & TSS200-Island & $1.09(0.45-2.66)$ & 0.853 \\
\hline cg24086405 & 0.679 & 0.707 & 0.728 & $1.38 \times 10^{-5}$ & 8 & DERL1 & TSS1500-Shore & $1.22(0.46-3.29)$ & 0.687 \\
\hline cg14299177 & 0.035 & 0.034 & 0.031 & $1.55 \times 10^{-5}$ & 8 & MTFR1 & 5'UTR-Shore & $1.1(0.44-2.7)$ & 0.844 \\
\hline cg02512123 & 0.825 & 0.843 & 0.849 & $1.61 \times 10^{-5}$ & 1 & ZC3H11A & 5'UTR-Shelf & $2.05(0.84-5.01)$ & 0.115 \\
\hline cg03828224 & 0.036 & 0.041 & 0.045 & $1.75 \times 10^{-5}$ & 5 & PPIC & TSS1500-Island & $0.95(0.37-2.44)$ & 0.910 \\
\hline cg16570133 & 0.08 & 0.09 & 0.098 & $1.85 \times 10^{-5}$ & 8 & N/A & IGR-Shore & $0.97(0.4-2.32)$ & 0.942 \\
\hline cg19614456 & 0.855 & 0.862 & 0.872 & $1.99 \times 10^{-5}$ & 1 & GABPB2 & 5'UTR-Open sea & $1.51(0.63-3.64)$ & 0.360 \\
\hline cg27362302 & 0.181 & 0.197 & 0.209 & $2.20 \times 10^{-5}$ & 18 & GNAL & Exon 1-Island & $1.14(0.46-2.82)$ & 0.779 \\
\hline cg21811204 & 0.396 & 0.429 & 0.44 & $2.31 \times 10^{-5}$ & 5 & SHROOM1 & Body-Island & $1.15(0.49-2.7)$ & 0.748 \\
\hline cg16432885 & 0.691 & 0.71 & 0.721 & $2.53 \times 10^{-5}$ & 3 & GSK3B & Body-Open sea & $3.66(1.33-10.11)$ & 0.012 \\
\hline cg00915676 & 0.651 & 0.663 & 0.678 & $2.95 \times 10^{-5}$ & 7 & $\mathrm{~N} / \mathrm{A}$ & IGR-Open sea & $3.24(1.12-9.4)$ & 0.030 \\
\hline cg10481023 & 0.753 & 0.774 & 0.783 & $3.01 \times 10^{-5}$ & 6 & GABRR2 & Body-Open sea & $1.31(0.54-3.19)$ & 0.546 \\
\hline cg15419054 & 0.087 & 0.081 & 0.073 & $3.15 \times 10^{-5}$ & 17 & NPEPPS & Body-Shore & $0.7(0.29-1.67)$ & 0.418 \\
\hline cg19078430 & 0.672 & 0.689 & 0.708 & $3.22 \times 10^{-5}$ & 17 & SSH2 & Body-Open sea & $0.89(0.38-2.11)$ & 0.797 \\
\hline cg10183781 & 0.719 & 0.733 & 0.756 & $3.65 \times 10^{-5}$ & 18 & АТР9B & Body-Open sea & $1.27(0.52-3.11)$ & 0.603 \\
\hline cg20171236 & 0.464 & 0.484 & 0.498 & $3.68 \times 10^{-5}$ & 4 & $\mathrm{~N} / \mathrm{A}$ & IGR-Open sea & $2.37(0.9-6.26)$ & 0.082 \\
\hline cg07160783 & 0.666 & 0.662 & 0.645 & $3.83 \times 10^{-5}$ & 16 & $\mathrm{~N} / \mathrm{A}$ & IGR-Open sea & $0.99(0.4-2.46)$ & 0.977 \\
\hline cg02747319 & 0.306 & 0.313 & 0.335 & $3.84 \times 10^{-5}$ & 2 & $\mathrm{~N} / \mathrm{A}$ & IGR-Shore & $2.01(0.81-5.02)$ & 0.134 \\
\hline cg07665241 & 0.7 & 0.723 & 0.736 & $3.89 \times 10^{-5}$ & 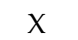 & CXorf36 & TSS1500-Open sea & $1.06(0.45-2.47)$ & 0.898 \\
\hline cg12058586 & 0.123 & 0.113 & 0.102 & $4.23 \times 10^{-5}$ & 12 & N/A & IGR-Open sea & $0.39(0.14-1.1)$ & 0.074 \\
\hline cg05135861 & 0.426 & 0.418 & 0.403 & $4.29 \times 10^{-5}$ & 18 & DLGAP1 & 5'UTR-Open sea & $0.72(0.28-1.88)$ & 0.503 \\
\hline cg00157515 & 0.079 & 0.087 & 0.1 & $4.32 \times 10^{-5}$ & 2 & LOC100132215 & TSS1500-Island & $2.06(0.77-5.52)$ & 0.151 \\
\hline cg26470340 & 0.02 & 0.023 & 0.027 & $4.33 \times 10^{-5}$ & 10 & ARHGAP21 & 5'UTR-Island & $1.02(0.4-2.61)$ & 0.965 \\
\hline cg11945022 & 0.576 & 0.594 & 0.642 & $4.51 \times 10^{-5}$ & 7 & DYNC1I1 & 5'UTR-Open sea & $0.62(0.25-1.55)$ & 0.308 \\
\hline cg18303466 & 0.753 & 0.76 & 0.776 & $4.57 \times 10^{-5}$ & 1 & SLC9A1 & TSS1500-Shore & $1.19(0.5-2.84)$ & 0.694 \\
\hline cg11650926 & 0.781 & 0.78 & 0.764 & $4.59 \times 10^{-5}$ & 5 & N/A & IGR-Open sea & $0.67(0.28-1.61)$ & 0.370 \\
\hline cg15954675 & 0.723 & 0.744 & 0.771 & $4.65 \times 10^{-5}$ & 3 & SYNPR & Body-Open sea & $3.11(1.08-8.95)$ & 0.036 \\
\hline cg25595028 & 0.842 & 0.852 & 0.86 & $4.72 \times 10^{-5}$ & 11 & RNF214 & Body-Open sea & $1.21(0.49-2.97)$ & 0.676 \\
\hline cg20981146 & 0.846 & 0.856 & 0.871 & $5.02 \times 10^{-5}$ & 1 & RSBN1 & Body-Open sea & $1.02(0.42-2.5)$ & 0.959 \\
\hline cg19734896 & 0.825 & 0.812 & 0.802 & $5.12 \times 10^{-5}$ & 1 & ILDR2 & Body-Shore & $0.55(0.22-1.36)$ & 0.196 \\
\hline cg12911208 & 0.744 & 0.731 & 0.719 & $5.13 \times 10^{-5}$ & 6 & RP11-73O6.4 & 5'UTR-Open sea & $0.89(0.36-2.19)$ & 0.793 \\
\hline cg13928649 & 0.13 & 0.138 & 0.156 & $5.21 \times 10^{-5}$ & 9 & PRDM12 & Body-Island & $1.35(0.51-3.55)$ & 0.546 \\
\hline cg05492453 & 0.101 & 0.087 & 0.082 & $5.32 \times 10^{-5}$ & 22 & FAM19A5 & Body-Open sea & $0.83(0.33-2.1)$ & 0.699 \\
\hline cg04492396 & 0.073 & 0.057 & 0.05 & $5.32 \times 10^{-5}$ & 8 & $\mathrm{~N} / \mathrm{A}$ & IGR-Open sea & $0.59(0.23-1.51)$ & 0.270 \\
\hline cg00290605 & 0.024 & 0.028 & 0.029 & $5.33 \times 10^{-5}$ & 22 & CTA-342B11.2 & TSS200-Island & $0.85(0.35-2.06)$ & 0.721 \\
\hline cg01382153 & 0.058 & 0.063 & 0.066 & $5.34 \times 10^{-5}$ & 2 & CCDC108 & Exon 1-Island & $1.11(0.44-2.79)$ & 0.822 \\
\hline cg00903099 & 0.131 & 0.144 & 0.15 & $5.37 \times 10^{-5}$ & 7 & HTR5A & TSS200-Shore & $1.14(0.47-2.78)$ & 0.774 \\
\hline cg18285105 & 0.631 & 0.652 & 0.677 & $5.37 \times 10^{-5}$ & 9 & RP11-87N24.3 & TSS1500-Open sea & $1.89(0.77-4.64)$ & 0.167 \\
\hline cg01049417 & 0.06 & 0.064 & 0.07 & $5.50 \times 10^{-5}$ & 3 & SCHIP1 & Body-Island & $1.3(0.5-3.39)$ & 0.595 \\
\hline cg07744841 & 0.191 & 0.208 & 0.221 & $5.62 \times 10^{-5}$ & 3 & SLC6A11 & TSS1500-Island & $3.5(1.21-10.17)$ & 0.021 \\
\hline cg14530623 & 0.308 & 0.326 & 0.343 & $5.66 \times 10^{-5}$ & 2 & ITSN2 & 5'UTR-Open sea & $1.7(0.69-4.22)$ & 0.250 \\
\hline cg25252658 & 0.623 & 0.644 & 0.67 & $5.67 \times 10^{-5}$ & 7 & FKBP6 & Body-Open sea & $2.08(0.83-5.24)$ & 0.120 \\
\hline cg03052956 & 0.252 & 0.268 & 0.306 & $5.70 \times 10^{-5}$ & $X$ & ARHGAP36 & TSS1500-Shore & $1.45(0.57-3.68)$ & 0.430 \\
\hline cg01909140 & 0.836 & 0.842 & 0.853 & $6.07 \times 10^{-5}$ & 9 & C9orf3 & Body-Open sea & $1.57(0.64-3.81)$ & 0.324 \\
\hline cg11179997 & 0.137 & 0.144 & 0.152 & $6.42 \times 10^{-5}$ & $X$ & $\mathrm{~N} / \mathrm{A}$ & IGR-Island & $0.85(0.34-2.17)$ & 0.737 \\
\hline cg13453374 & 0.222 & 0.236 & 0.261 & $6.44 \times 10^{-5}$ & 3 & RP11-649A16.1 & 3'UTR-Open sea & $1.15(0.48-2.78)$ & 0.753 \\
\hline cg21081034 & 0.177 & 0.192 & 0.203 & $6.57 \times 10^{-5}$ & 2 & LOC100132215 & TSS1500-Island & $0.73(0.27-1.98)$ & 0.532 \\
\hline cg16372976 & 0.494 & 0.531 & 0.57 & $6.74 \times 10^{-5}$ & 1 & RFWD2 & Body-Shelf & $1.11(0.45-2.76)$ & 0.824 \\
\hline cg00088299 & 0.013 & 0.016 & 0.018 & $6.80 \times 10^{-5}$ & 4 & N/A & IGR-Island & $2.37(0.89-6.32)$ & 0.084 \\
\hline cg25339368 & 0.809 & 0.817 & 0.828 & $6.92 \times 10^{-5}$ & 17 & TBCD & Body-Shore & $1.22(0.49-3.06)$ & 0.673 \\
\hline cg25215047 & 0.804 & 0.807 & 0.837 & $6.97 \times 10^{-5}$ & 13 & $\mathrm{~N} / \mathrm{A}$ & IGR-Open sea & $1.29(0.54-3.07)$ & 0.565 \\
\hline cg07629204 & 0.484 & 0.507 & 0.534 & $7.14 \times 10^{-5}$ & 10 & $\mathrm{~N} / \mathrm{A}$ & IGR-Open sea & $2.41(0.8-7.23)$ & 0.118 \\
\hline cg21377071 & 0.495 & 0.521 & 0.541 & $7.31 \times 10^{-5}$ & 15 & ADAMTSL3 & 5'UTR-Shore & $1.94(0.77-4.89)$ & 0.160 \\
\hline cg23099959 & 0.622 & 0.643 & 0.665 & $7.54 \times 10^{-5}$ & 15 & RP11-66B24.2 & 5'UTR-Shore & $0.6(0.22-1.61)$ & 0.309 \\
\hline
\end{tabular}


Table 2. Cont.

\begin{tabular}{|c|c|c|c|c|c|c|c|c|c|}
\hline \multirow[b]{2}{*}{ CpG ID } & \multicolumn{3}{|c|}{$\beta$ Value } & \multirow[b]{2}{*}{$\begin{array}{l}P \text { for } \\
\text { Trend }\end{array}$} & \multirow[b]{2}{*}{ Chr. } & \multirow[b]{2}{*}{ Gene } & \multirow[b]{2}{*}{ CpG Location } & \multicolumn{2}{|c|}{ Cox Analysis for BCR } \\
\hline & $\mathrm{GS}=6$ & $\mathrm{GS}=7$ & $\mathrm{GS} \geq 8$ & & & & & HR $(95 \%$ CI $)$ & $\begin{array}{c}p \\
\text { Value }\end{array}$ \\
\hline cg14824107 & 0.73 & 0.721 & 0.683 & $7.58 \times 10^{-5}$ & 17 & TBCD & Body-Shore & $0.63(0.25-1.59)$ & 0.326 \\
\hline cg01993946 & 0.699 & 0.688 & 0.671 & $8.00 \times 10^{-5}$ & 10 & $\mathrm{~N} / \mathrm{A}$ & IGR-Open sea & $0.52(0.21-1.28)$ & 0.156 \\
\hline cg11875624 & 0.06 & 0.084 & 0.083 & $8.08 \times 10^{-5}$ & $X$ & FGF13 & 5'UTR-Island & $0.93(0.36-2.41)$ & 0.882 \\
\hline cg19004134 & 0.02 & 0.021 & 0.024 & $8.12 \times 10^{-5}$ & 6 & VTA1 & TSS200-Island & $1.58(0.65-3.82)$ & 0.310 \\
\hline cg03854198 & 0.087 & 0.093 & 0.104 & $8.14 \times 10^{-5}$ & 12 & NTF3 & Exon 1-Island & $1.49(0.53-4.15)$ & 0.448 \\
\hline cg13786089 & 0.092 & 0.103 & 0.117 & $8.28 \times 10^{-5}$ & 19 & ZFR2 & TSS200-Island & $1.68(0.67-4.26)$ & 0.271 \\
\hline cg05917797 & 0.775 & 0.782 & 0.792 & $8.40 \times 10^{-5}$ & 14 & $\mathrm{~N} / \mathrm{A}$ & IGR-Open sea & $1.58(0.64-3.87)$ & 0.318 \\
\hline cg12574406 & 0.813 & 0.822 & 0.834 & $8.60 \times 10^{-5}$ & 8 & $\mathrm{~N} / \mathrm{A}$ & IGR-Open sea & $1.2(0.48-3.04)$ & 0.693 \\
\hline cg04855249 & 0.788 & 0.782 & 0.77 & $8.97 \times 10^{-5}$ & 15 & OCA2 & Body-Open sea & $0.92(0.37-2.29)$ & 0.859 \\
\hline cg23409289 & 0.018 & 0.02 & 0.023 & $9.42 \times 10^{-5}$ & 5 & C5orf56 & TSS1500-Island & $1.46(0.59-3.62)$ & 0.410 \\
\hline cg12949141 & 0.593 & 0.612 & 0.635 & $9.48 \times 10^{-5}$ & 5 & PCBD2 & Body-Open sea & $1.56(0.61-3.98)$ & 0.354 \\
\hline cg00170540 & 0.768 & 0.785 & 0.791 & $9.55 \times 10^{-5}$ & 16 & HSDL1 & $5^{\prime}$ UTR-Open sea & $1.18(0.49-2.79)$ & 0.715 \\
\hline cg22851420 & 0.22 & 0.238 & 0.25 & $9.58 \times 10^{-5}$ & 1 & HPCAL4 & Body-Island & $0.67(0.26-1.68)$ & 0.391 \\
\hline cg04921989 & 0.07 & 0.077 & 0.084 & $9.84 \times 10^{-5}$ & 2 & N/A & IGR-Island & $1.23(0.51-2.95)$ & 0.651 \\
\hline cg13551368 & 0.508 & 0.497 & 0.484 & $9.87 \times 10^{-5}$ & 5 & $\mathrm{~N} / \mathrm{A}$ & IGR-Open sea & $0.5(0.19-1.3)$ & 0.154 \\
\hline cg06465285 & 0.776 & 0.796 & 0.815 & $9.95 \times 10^{-5}$ & 8 & $\mathrm{~N} / \mathrm{A}$ & IGR-Open sea & $0.79(0.32-1.94)$ & 0.608 \\
\hline
\end{tabular}

To test the prognostic value of these DMCs, we used a multivariable Cox proportional hazards model to determine the associations of these DMCs with biochemical recurrence (BCR). Among these top DMCs, only two were independently associated with BCR at a significance level of 0.05 (Table 2). High methylation at cg16432885 and cg00915676 was associated with significantly increased risks of $\mathrm{BCR}(\mathrm{HR}=3.66,95 \% \mathrm{CI}, 1.33-10.11, \mathrm{P}=0.012$ and $3.24,95 \% \mathrm{CI}, 1.12-9.4, \mathrm{P}=0.030$, respectively). The association of high methylation with worse prognosis is consistent with increased methylation level in patients with higher Gleason score at these two CpG sites.

\subsection{Differentially Methylated Regions/Genes (DMRs) in High-Grade African American PCa Patients}

Due to the fact that DMRs are more likely to be functionally important than scattered individual DMCs, we next searched for DMRs in which at least seven CpGs showed consistently increased or decreased methylation associated with increasing Gleason scores. A total of 77 DMRs were found (Table 3 and Supplemental Table S1). There were 10 homeobox genes (ALX1, HOXC11, HOXD1, HOXD8, HOXD11, LHX8, MSX2, NKX6-2, PAX7, POU4F2) and six zinc finger protein genes (ZBTB16, ZNF83, ZNF471, ZNF577, ZNF714, ZSCAN1). Figure 2 shows the representative DMRs. The majority of these DMRs were found in gene promoter regions with higher methylation levels in patients with higher Gleason scores. We performed a gene ontology (GO) molecular pathway enrichment analysis of these 77 DMRs. The main enriched pathway was DNA-binding transcriptional factor activity, consistent with the major functional roles of homeobox proteins and zinc finger proteins as transcriptional factors.

Table 3. Differentially methylated genes between different Gleason scores.

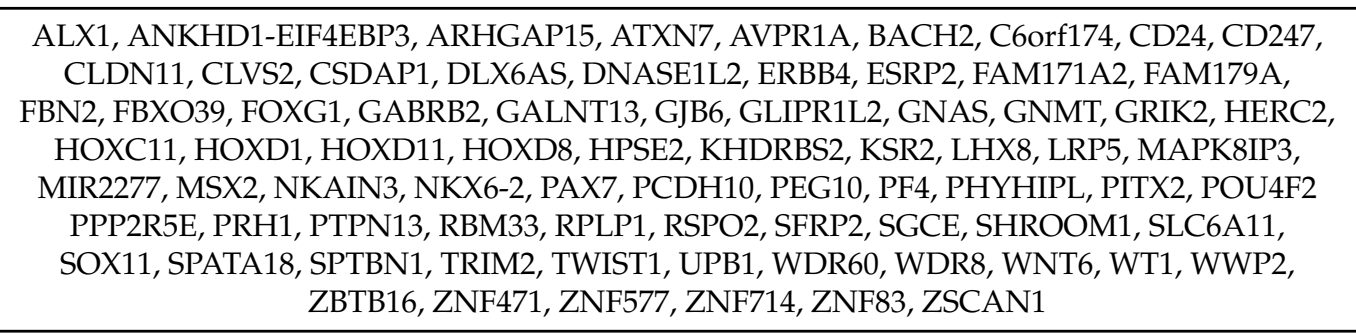




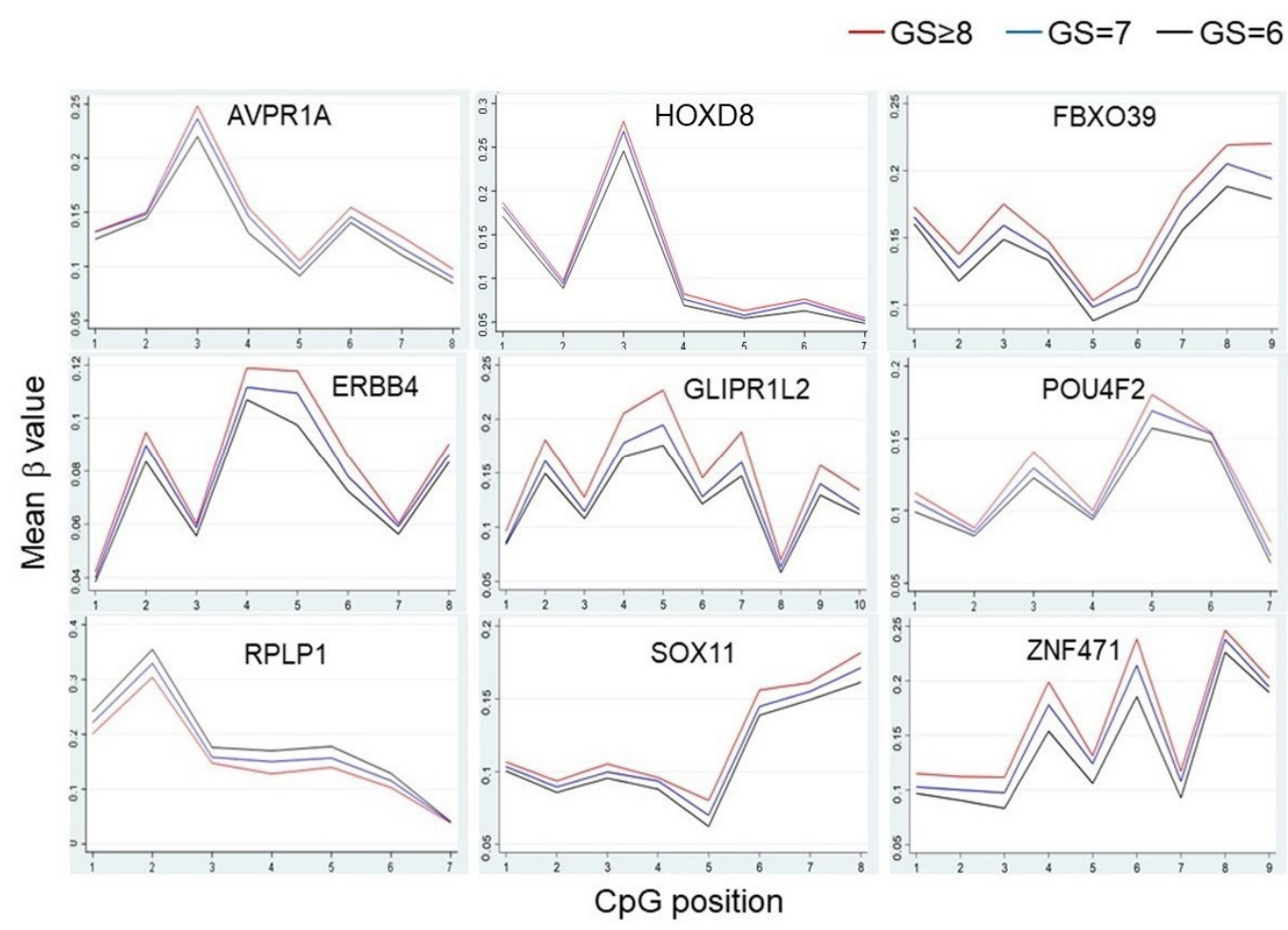

Figure 2. Representative differentially methylated genes between African American prostate cancer patients with high and low Gleason scores.

\section{Discussion}

In this study, we performed genome-wide $\mathrm{CpG}$ methylation profiling of leukocyte DNA from 280 African American PCa patients with different Gleason scores to identify intrinsic methylation differences that may serve as predictors of aggressive PCa in African Americans. To our knowledge, this is the first EWAS of PCa in African Americans.

As expected, the mean methylation level was the lowest in the core promoter region (TSS-200) (mean $\beta$ value $<0.2$ ) and also remained at low levels in Exon 1, TSS-1500, and $5^{\prime}$ UTR (mean $\beta$ values range between 0.2 and 0.4 ), but much higher levels were found in the gene body, $3^{\prime}$ UTR, and IGR (mean $\beta$ values range between 0.5 and 0.7 ), consistent with the literature of lower promoter methylation allowing a more open chromatin structure and active transcription [28]. When we compared the overall leukocyte methylation levels of GS $=6, \mathrm{GS}=7$, and GS $\geq 8$ patients, there was a slight increase of methylation level with the increase of Gleason scores. We identified a panel of promising DMCs that are differentially methylated between different Gleason scores. These CpG site methylations may serve as biomarkers for aggressive cancer. More importantly, we identified at least 77 DMRs associated with high-grade PCa. The majority of these DMRs exhibited increased methylation with increased Gleason scores and are located in the promoter island regions of functionally important genes, indicating an overall downregulation of gene expression in leukocytes of high-grade PCa patients, likely affecting immune response, DNA repair, etc., as well as contributing to the aggressive phenotypes.

Among the DMRs between PCa patients with high and low Gleason scores, there were 10 homeobox genes and six zinc finger genes. At least 235 homeobox genes have been identified in the human genome [29]. Homeobox genes contain a highly conserved DNA sequence of about $180 \mathrm{bp}$ encoding the homeodomain of 60 amino acids. Homeodomain proteins act as transcription factors that specifically bind to DNA motifs and regulate the expression of numerous target genes involved in cell proliferation, apoptosis, adhesion, angiogenesis, and DNA repair $[30,31]$. Homeobox genes are frequently dysregulated in hematological malignancies and solid tumors [31,32]. In addition, homeodomain proteins also play important roles in regulating inflammation and immune response [33-35]. Aber- 
rant DNA methylation is one of the major causes of homeobox gene dysregulation during cancer development and progression [36]. Many homeobox genes are hypermethylated in various cancers, including prostate cancer [36]. Two of the identified homeobox genes in our current study, HODX1 and HOXD8, have been shown to play a tumor-suppressor function in various cancers [37-39]. Increased methylation of HOXD8 was observed in lymphoma patients compared to normal B cells [40]. Hypermethylation of HOXD8 in urine was associated with disease progression in PCa patients on active surveillance [41]. HOXD8 and several other homeobox genes were hypermethylated in aged muscle tissue compared with young tissue [42]. These previous reports are consistent with our observations of increased HOXD8 methylation in high Gleason score PCa patients. Increased methylation of homeobox genes in leukocyte DNA of PCa patients with high Gleason scores may indicate weakened immune response and suboptimal DNA repair capacity.

The zinc finger proteins are the largest family of transcriptional factors, which function through the binding of the zinc finger domain to specific DNA sequences [43]. In addition to DNA binding, zinc finger proteins can also bind to RNAs and proteins [44]. Zinc finger proteins play crucial roles in transcriptional and post-transcriptional regulation of immune response [45] and are involved in cancer development and progression [46]. Among the identified zinc finger proteins in our study, ZNF471 functions as a tumor suppressor in several cancers and is frequently hypermethylated in tumor tissues [47-51]. Higher levels of ZNF577 methylation in leukocytes have been associated with obesity [52]. As obesity is associated with aggressive PCa [53], ZNF577 methylation may provide a biological link between obesity and PCa progression. Another study showed higher ZNF577 methylation in T-cells from kidney transplant patients who developed de novo skin cancer than those who did not develop skin cancer [54], suggesting increased methylation of ZNF577 may lead to weakened immune response. The exact molecular mechanisms for the roles of these homeodomain and zinc finger proteins play in aggressive PCa warrant further investigation.

Another interesting DMR in our study is SOX11. SOX proteins are a family of about 20 transcriptional factors that have a conserved high-mobility group (HMG) domain that mediates DNA binding [55]. SOX11 has been shown to act as a tumor suppressor in several cancers, including prostate cancer [56,57]. SOX11 is hypermethylated in prostate tumor tissues and the hypermethylation is associated with aggressive clinical features, including higher PSA and Gleason scores [58]. A previous study showed higher SOX11 methylation in leukocyte DNA from gastric patients than that from controls [59]. Our study is the first to show an increased methylation of SOX11 in leukocyte DNA from aggressive PCa patients.

Previously, we performed a similar study of EWAS in European American (EA) prostate cancer patients using the Illumina 450k methylation arrays [23], which covered about $60 \%$ of the CpG sites on the MethylationEPIC arrays in this current study. The overall methylation level (mean $\beta$ value) is higher in AA than EA patients with the same Gleason scores. There were more hypermethylated than hypomethylated $\mathrm{CpG}$ sites in patients with higher Gleason scores compared to those with lower Gleason scores. There was very little overlap among the top DMCs and DMRs, but there was significant enrichment of transcriptional factors among DMRs.

CpG methylation in leukocyte DNA sits at the interface between genetics and environment, with longstanding effects on gene expression, inflammation, and immune response [60]. Leukocyte DNA methylation signatures are excellent biomarkers of age and smoking status in a normal population [61-64]. We compared methylation patterns in PCa patients with different Gleason scores. The age and smoking status distributions in patients with GS6, GS7, and GS $\geq 8$ in our study were very similar. In addition, most of our patients were never smokers. The differences of $\mathrm{CpG}$ site methylation were consistent when we performed stratified analyses by age group and smoking status (data not shown). The top DMCs in our study (Table 2) were not among those DMCs found in the aging and smoking methylation signatures [61-64]. Therefore, the DMCs identified in this current study were not likely due to age, smoking, or other potential confounders. Leukocyte DNA 
methylation profiles have been used to derive systemic inflammation indices [65-68] and immune cell lineages [60]. We did not observe significant differences in the immune cell subpopulations between patients with different Gleason scores (data not shown), indicating that the differential methylations were not due to differences in immune cell subtypes, but were intrinsic biological changes associated with disease severity across various immune cell types. Leukocyte DNA methylation levels, therefore, may be valuable biomarkers for aggressive PCa patients. However, it is worth noting that the absolute methylation difference ( $\beta$ value difference) at each $C p G$ site between high-grade and low-grade patients was small (generally $<0.05$ ). This small difference of leukocyte DNA methylation level between high-grade and low-grade PCa patients is not surprising because of the high background of normal leukocyte methylation.

There were some limitations of this study. Firstly, this was a single-center study. External validations using independent populations are warranted to confirm the DMCs and DMRs identified from this study. Secondly, we only reported nominal significance values (Table 2). None of the DMCs reached genome-wide significance level $(\mathrm{P}<5.9$ $\times 10^{-8}$ ) after correcting for multiple testing of $850 \mathrm{~K}$ markers. This is not surprising as an extremely large sample size is always required for genome-wide association studies (GWASs) and EWASs to reach genome-wide significance level. Future large collaborative efforts are needed to significantly increase the sample size and bring the top DMCs to a genome-wide significance level.

In summary, we performed genome-wide DNA methylation profiling of leukocyte DNA in African American PCa patients. We observed slightly increased overall DNA methylation in high-grade PCa patients compared to low-grade PCa patients. We identified a large panel of differentially methylated $\mathrm{CpG}$ sites between patients with different Gleason scores. We found 77 differentially methylated genes between high-grade and low-grade patients, and homeodomain protein and zinc finger protein genes were enriched in DMRs. Our study suggests that leukocyte DNA methylation may be a valuable biomarker for aggressive PCa and the identified DMRs provide biological insights into the modulation of immune response by aggressive $\mathrm{PCa}$.

Supplementary Materials: The following are available online at https:/ / www.mdpi.com/article/ 10.3390/biom11121826/s1, Table S1: Differentially methylated regions/genes and their CpG site methylations between different Gleason scores in African American prostate cancer patients.

Author Contributions: Conceptualization, Y.X., C.-W.T., W.-S.C., D.-T.B. and J.G.; data curation, Y.X., C.-W.T., W.-S.C., M.H. and J.G.; formal analysis, Y.H., M.H. and J.G.; funding acquisition, J.G.; investigation, Y.X., C.-W.T., W.-S.C., Y.H., D.-T.B. and J.G.; methodology, Y.X., C.-W.T., W.-S.C., Y.H., C.A.P., D.-T.B. and J.G.; project administration, J.G.; resources, C.A.P. and J.G.; supervision, J.G.; writing—original draft, Y.X., C.-W.T., W.-S.C., D.-T.B. and J.G.; data curation, Y.X., C.-W.T., W.-S.C., M.H. and J.G.; writing-review and editing, Y.X., C.-W.T., W.-S.C., Y.H., M.H., C.A.P., D.-T.B. and J.G. All authors have read and agreed to the published version of the manuscript.

Funding: This research was funded by a Cancer Prevention and Research Institute of Texas (CPRIT) grant (grant number: RP140556) and a seed grant from The University of Texas MD Anderson Cancer Center Duncan Family Institute for Cancer Prevention and Risk Assessment.

Institutional Review Board Statement: The study was conducted according to the guidelines of the Declaration of Helsinki and approved by the Institutional Review Board of the University of Texas MD Anderson Cancer Center (protocol number PA12-0547, approved on 13 July 2021).

Informed Consent Statement: Informed consent was obtained from all subjects involved in the study.

Data Availability Statement: The data presented in this study are available on request from the corresponding author. The data are not publicly available due to due to privacy information and ethical consideration. 
Acknowledgments: We thank the Biospecimen Extraction Facility of MD Anderson Cancer Center for DNA extraction and the Population Genetics Core of MD Anderson Cancer Center for performing methylation arrays.

Conflicts of Interest: The authors declare no conflict of interest. The funders had no role in the design of the study; in the collection, analyses, or interpretation of data; in the writing of the manuscript; or in the decision to publish the results.

\section{References}

1. Siegel, R.L.; Miller, K.D.; Fuchs, H.E.; Jemal, A. Cancer Statistics, 2021. CA Cancer J. Clin. 2021, 71, 7-33. [CrossRef] [PubMed]

2. McGinley, K.F.; Tay, K.J.; Moul, J.W. Prostate cancer in men of African origin. Nat. Rev. Urol. 2016, 13, 99-107. [CrossRef]

3. Rebbeck, T.R. Prostate Cancer Disparities by Race and Ethnicity: From Nucleotide to Neighborhood. Cold Spring Harb. Perspect. Med. 2018, 8, a030387. [CrossRef] [PubMed]

4. Kan, Z.; Jaiswal, B.S.; Stinson, J.; Janakiraman, V.; Bhatt, D.; Stern, H.M.; Yue, P.; Haverty, P.M.; Bourgon, R.; Zheng, J.; et al. Diverse somatic mutation patterns and pathway alterations in human cancers. Nat. Cell Biol. 2010, 466, 869-873. [CrossRef]

5. Vogelstein, B.; Papadopoulos, N.; Velculescu, V.E.; Zhou, S.; Diaz, L.A., Jr.; Kinzler, K.W. Cancer Genome Landscapes. Science 2013, 339, 1546-1558. [CrossRef]

6. Fraser, M.; Sabelnykova, V.Y.; Yamaguchi, T.; Heisler, L.; Livingstone, J.; Huang, V.; Shiah, Y.-J.; Yousif, F.; Lin, X.; Masella, A.P.; et al. Genomic hallmarks of localized, non-indolent prostate cancer. Nat. Cell Biol. 2017, 541, 359-364. [CrossRef] [PubMed]

7. Blattner, M.; Lee, D.J.; O’Reilly, C.; Park, K.; MacDonald, T.Y.; Khani, F.; Turner, K.R.; Chiu, Y.-L.; Wild, P.J.; Dolgalev, I.; et al. SPOP mutations in prostate cancer across demographically diverse patient cohorts. Neoplasia 2014, 16, 14-20. [CrossRef]

8. Goering, W.; Kloth, M.; Schulz, W.A. DNA Methylation Changes in Prostate Cancer. Springer Protocols Handbooks 2012, 863, 47-66. [CrossRef]

9. Jerónimo, C.; Bastian, P.J.; Bjartell, A.; Carbone, G.M.; Catto, J.; Clark, S.; Henrique, R.; Nelson, W.G.; Shariat, S.F. Epigenetics in Prostate Cancer: Biologic and Clinical Relevance. Eur. Urol. 2011, 60, 753-766. [CrossRef]

10. Boström, P.J.; Bjartell, A.S.; Catto, J.; Eggener, S.E.; Lilja, H.; Loeb, S.; Schalken, J.; Schlomm, T.; Cooperberg, M.R. Genomic Predictors of Outcome in Prostate Cancer. Eur. Urol. 2015, 68, 1033-1044. [CrossRef]

11. Van Neste, L.; Partin, A.W.; Stewart, G.; Epstein, J.I.; Harrison, D.; Van Criekinge, W. Risk score predicts high-grade prostate cancer in DNA-methylation positive, histopathologically negative biopsies. Prostate 2016, 76, 1078-1087. [CrossRef]

12. Waterhouse, R.L.; Van Neste, L.; Moses, K.A.; Barnswell, C.; Silberstein, J.L.; Jalkut, M.; Tutrone, R.; Sylora, J.; Anglade, R.; Murdock, M.; et al. Evaluation of an Epigenetic Assay for Predicting Repeat Prostate Biopsy Outcome in African American Men. Urology 2019, 128, 62-65. [CrossRef]

13. Flanagan, J.M.; Wilson, A.; Koo, C.; Masrour, N.; Gallon, J.; Loomis, E.; Flower, K.; Wilhelm-Benartzi, C.; Hergovich, A.; Cunnea, P.; et al. Platinum-Based Chemotherapy Induces Methylation Changes in Blood DNA Associated with Overall Survival in Patients with Ovarian Cancer. Clin. Cancer Res. 2016, 23, 2213-2222. [CrossRef] [PubMed]

14. Joo, J.E.; Fab, K.C.; Dowty, J.G.; Milne, R.L.; Wong, E.M.; Dugue, P.-A.; English, D.; Hopper, J.L.; Goldgar, D.E.; Giles, G.G.; et al. Heritable DNA methylation marks associated with susceptibility to breast cancer. Nat. Commun. 2018, 9, 1-12. [CrossRef]

15. Dugué, P.-A.; Dowty, J.G.; Joo, J.E.; Wong, E.M.; Makalic, E.; Schmidt, D.F.; English, D.R.; Hopper, J.L.; Pedersen, J.; Severi, G.; et al. Heritable methylation marks associated with breast and prostate cancer risk. Prostate 2018, 78, 962-969. [CrossRef]

16. Barry, K.H.; Moore, L.E.; Sampson, J.N.; Koutros, S.; Yan, L.; Meyer, A.; Reddy, M.; Oler, A.J.; Cook, M.B.; Jr, J.F.F.; et al. Prospective study of DNA methylation at chromosome 8q24 in peripheral blood and prostate cancer risk. Br. J. Cancer 2017, 116, 1470-1479. [CrossRef] [PubMed]

17. Han, Y.; Xu, J.; Kim, J.; Wu, X.; Gu, J. Methylation of subtelomeric repeat D4Z4 in peripheral blood leukocytes is associated with biochemical recurrence in localized prostate cancer patients. Carcinoenesis 2017, 38, 821-826. [CrossRef]

18. Han, Y.; Xu, J.; Kim, J.; Wu, X.; Gu, J. LINE-1 methylation in peripheral blood leukocytes and clinical characteristics and prognosis of prostate cancer patients. Oncotarget 2017, 8, 94020-94027. [CrossRef]

19. Xu, J.; Tsai, C.-W.; Chang, W.-S.; Han, Y.; Bau, D.-T.; Pettaway, C.A.; Gu, J. Methylation of global DNA repeat LINE-1 and subtelomeric DNA repeats D4Z4 in leukocytes is associated with biochemical recurrence in African American prostate cancer patients. Carcinog. 2019, 40, 1055-1060. [CrossRef]

20. Moses-Fynn, E.; Tang, W.; Beyene, D.; Apprey, V.; Copeland, R.; Kanaan, Y.; Kwabi-Addo, B. Correlating blood-based DNA methylation markers and prostate cancer risk in African-American men. PLoS ONE 2018, 13, e0203322. [CrossRef]

21. FitzGerald, L.M.; Naeem, H.; Makalic, E.; Schmidt, D.F.; Dowty, J.G.; Joo, J.E.; Jung, C.-H.; Bassett, J.K.; Dugue, P.-A.; Chung, J.; et al. Genome-Wide Measures of Peripheral Blood Dna Methylation and Prostate Cancer Risk in a Prospective Nested Case-Control Study. Prostate 2017, 77, 471-478. [CrossRef]

22. Mehdi, A.; Cheishvili, D.; Arakelian, A.; Bismar, T.A.; Szyf, M.; Rabbani, S.A. DNA methylation signatures of Prostate Cancer in peripheral T-cells. BMC Cancer 2020, 20, 1-11. [CrossRef]

23. Han, Y.; Zhang, M.; Xu, J.; Li, J.; Xu, Y.; Thompson, T.C.; Logothetis, C.J.; Sun, D.; Gu, J. Genome-wide DNA methylation profiling of leukocytes identifies CpG methylation signatures of aggressive prostate cancer. Am. J. Cancer Res. 2021, 11, 968-978. 
24. Pidsley, R.; Zotenko, E.; Peters, T.J.; Lawrence, M.G.; Risbridger, G.P.; Molloy, P.; Van Djik, S.; Muhlhausler, B.; Stirzaker, C.; Clark, S.J. Critical evaluation of the Illumina MethylationEPIC BeadChip microarray for whole-genome DNA methylation profiling. Genome Biol. 2016, 17, 208. [CrossRef]

25. Aryee, M.J.; Jaffe, A.E.; Corrada-Bravo, H.; Ladd-Acosta, C.; Feinberg, A.P.; Hansen, K.D.; Irizarry, R.A. Minfi: A flexible and comprehensive Bioconductor package for the analysis of Infinium DNA methylation microarrays. Bioinformatics 2014, 30 1363-1369. [CrossRef] [PubMed]

26. Leek, J.T.; Johnson, W.; Parker, H.S.; Jaffe, A.; Storey, J. The sva package for removing batch effects and other unwanted variation in high-throughput experiments. Bioinformatics 2012, 28, 882-883. [CrossRef]

27. Jaffe, A.E.; Irizarry, R.A. Accounting for cellular heterogeneity is critical in epigenome-wide association studies. Genome Biol. 2014, 15, R31. [CrossRef]

28. Zhu, H.; Wang, G.; Qian, G.W.J. Transcription factors as readers and effectors of DNA methylation. Nat. Rev. Genet. 2016, 17, 551-565. [CrossRef] [PubMed]

29. Holland, P.W.H.; Booth, H.A.F.; Bruford, E. Classification and nomenclature of all human homeobox genes. BMC Biol. 2007, 5, 1-28. [CrossRef]

30. Bürglin, T.R.; Affolter, M. Homeodomain proteins: An update. Chromosoma 2016, 125, 497-521. [CrossRef] [PubMed]

31. Haria, D.; Naora, H. Homeobox Gene Deregulation: Impact on the Hallmarks of Cancer. Cancer Hallm. 2013, 1, 67-76. [CrossRef]

32. Shah, N.; Sukumar, S. The Hox genes and their roles in oncogenesis. Nat. Rev. Cancer 2010, 10, 361-371. [CrossRef]

33. Pai, P.; Sukumar, S. HOX genes and the NF- $\mathrm{BB}$ pathway: A convergence of developmental biology, inflammation and cancer biology. Biochim. Biophys. Acta (BBA) Bioenerg. 2020, 1874, 188450. [CrossRef]

34. Northcott, J.M.; Northey, J.J.; Barnes, J.M.; Weaver, V.M. Fighting the force: Potential of homeobox genes for tumor microenvironment regulation. Biochim. Biophys. Acta (BBA) Bioenerg. 2015, 1855, 248-253. [CrossRef]

35. Scott, C.L.; Omilusik, K.D. ZEBs: Novel Players in Immune Cell Development and Function. Trends Immunol. 2019, 40, 431-446. [CrossRef] [PubMed]

36. Rodrigues, M.F.S.D.; Esteves, C.M.; Xavier, F.C.A.; Nunes, F.D. Methylation status of homeobox genes in common human cancers. Genomics 2016, 108, 185-193. [CrossRef]

37. Cui, Y.; Zhang, C.; Li, Y.; Ma, S.; Cao, W.; Guan, F. HOXD1 functions as a novel tumor suppressor in kidney renal clear cell carcinoma. Cell Biol. Int. 2021, 45, 1246-1259. [CrossRef] [PubMed]

38. Mansour, M.A.; Senga, T. HOXD8 exerts a tumor-suppressing role in colorectal cancer as an apoptotic inducer. Int. J. Biochem. Cell Biol. 2017, 88, 1-13. [CrossRef] [PubMed]

39. Zhang, Y.; Yu, Y.; Su, X.; Lu, Y. HOXD8 inhibits the proliferation and migration of triple-negative breast cancer cells and induces apoptosis in them through regulation of AKT/mTOR pathway. Reprod. Biol. 2021, 21, 100544. [CrossRef] [PubMed]

40. Leshchenko, V.V.; Kuo, P.-Y.; Shaknovich, R.; Yang, D.T.; Gellen, T.; Petrich, A.M.; Yu, Y.; Remache, Y.; Weniger, M.A.; Rafiq, S.; et al. Genomewide DNA methylation analysis reveals novel targets for drug development in mantle cell lymphoma. Blood 2010, 116, 1025-1034. [CrossRef]

41. Zhao, F.; Olkhov-Mitsel, E.; Van der Kwast, T.; Sykes, J.; Zdravic, D.; Venkateswaran, V.; Zlotta, A.R.; Loblaw, A.; Fleshner, N.E.; Klotz, L.; et al. Urinary DNA Methylation Biomarkers for Noninvasive Prediction of Aggressive Disease in Patients with Prostate Cancer on Active Surveillance. J. Urol. 2017, 197, 335-341. [CrossRef]

42. Turner, D.C.; Gorski, P.P.; Maasar, M.F.; Seaborne, R.A.; Baumert, P.; Brown, A.D.; Kitchen, M.O.; Erskine, R.M.; Dos-Remedios, I.; Voisin, S.; et al. DNA methylation across the genome in aged human skeletal muscle tissue and muscle-derived cells: The role of HOX genes and physical activity. Sci. Rep. 2020, 10, 1-19. [CrossRef] [PubMed]

43. Krishna, S.S.; Majumdar, I.; Grishin, N.V. Structural classification of zinc fingers: SURVEY AND SUMMARY. Nucleic Acids Res. 2003, 31, 532-550. [CrossRef]

44. Cassandri, M.; Smirnov, A.; Novelli, F.; Pitolli, C.; Agostini, M.; Malewicz, M.; Melino, G.; Raschellà, G. Zinc-finger proteins in health and disease. Cell Death Discov. 2017, 3, 17071. [CrossRef]

45. Rakhra, G. Zinc finger proteins: Insights into the transcriptional and post transcriptional regulation of immune response. Mol. Biol. Rep. 2021, 48, 5735-5743. [CrossRef]

46. Jen, J.; Wang, Y.-C. Zinc finger proteins in cancer progression. J. Biomed. Sci. 2016, 23, 1-9. [CrossRef] [PubMed]

47. Cao, L.; Wang, S.; Zhang, Y.; Wong, K.-C.; Nakatsu, G.; Wang, X.; Wong, S.; Ji, J.; Yu, J. Zinc-finger protein 471 suppresses gastric cancer through transcriptionally repressing downstream oncogenic PLS3 and TFAP2A. Oncogene 2018, 37, 3601-3616. [CrossRef]

48. Sun, R.; Xiang, T.; Tang, J.; Peng, W.; Luo, J.; Li, L.; Qiu, Z.; Tan, Y.; Ye, L.; Zhang, M.; et al. 19q13 KRAB zinc-finger protein ZNF471 activates MAPK10/JNK3 signaling but is frequently silenced by promoter CpG methylation in esophageal cancer. Theranostics 2020, 10, 2243-2259. [CrossRef]

49. Tao, C.; Luo, J.; Tang, J.; Zhou, D.; Feng, S.; Qiu, Z.; Putti, T.C.; Xiang, T.; Tao, Q.; Li, L.; et al. The tumor suppressor Zinc finger protein 471 suppresses breast cancer growth and metastasis through inhibiting AKT and Wnt/ $\beta$-catenin signaling. Clin. Epigenet. 2020, 12, 173. [CrossRef] [PubMed]

50. Bhat, S.; Kabekkodu, S.P.; Adiga, D.; Fernandes, R.; Shukla, V.; Bhandari, P.; Pandey, D.; Sharan, K.; Satyamoorthy, K. ZNF471 modulates EMT and functions as methylation regulated tumor suppressor with diagnostic and prognostic significance in cervical cancer. Cell Biol. Toxicol. 2021, 37, 731-749. [CrossRef] 
51. Bhat, S.; Kabekkodu, S.; Jayaprakash, C.; Radhakrishnan, R.; Ray, S.; Satyamoorthy, K. Gene promoter-associated CpG island hypermethylation in squamous cell carcinoma of the tongue. Virchows Archiv. 2017, 470, 445-454. [CrossRef]

52. Lorenzo, P.M.; Izquierdo, A.G.; Diaz-Lagares, A.; Carreira, M.C.; Macias-Gonzalez, M.; Sandoval, J.; Cueva, J.; Lopez-Lopez, R.; Casanueva, F.F.; Crujeiras, A.B. ZNF577 Methylation Levels in Leukocytes From Women With Breast Cancer Is Modulated by Adiposity, Menopausal State, and the Mediterranean Diet. Front. Endocrinol. 2020, 11, 245. [CrossRef]

53. Allott, E.H.; Masko, E.M.; Freedland, S.J. Obesity and Prostate Cancer: Weighing the Evidence. Eur. Urol. 2013, 63, 800-809. [CrossRef]

54. Peters, F.S.; Peeters, A.M.A.; Mandaviya, P.R.; Van Meurs, J.B.J.; Hofland, L.J.; Van De Wetering, J.; Betjes, M.G.H.; Baan, C.C.; Boer, K. Differentially methylated regions in T cells identify kidney transplant patients at risk for de novo skin cancer. Clin. Epigenet. 2018, 10, 81. [CrossRef]

55. Kamachi, Y.; Kondoh, H. Sox proteins: Regulators of cell fate specification and differentiation. Development 2013, 140, 4129-4144. [CrossRef] [PubMed]

56. Yang, Z.; Jiang, S.; Lu, C.; Ji, T.; Yang, W.; Li, T.; Lv, J.; Hu, W.; Yang, Y.; Jin, Z. SOX11: Friend or foe in tumor prevention and carcinogenesis? Ther. Adv. Med Oncol. 2019, 11, 1758835919853449. [CrossRef] [PubMed]

57. Yao, Z.; Sun, B.; Hong, Q.; Yan, J.; Mu, D.; Li, J.; Sheng, H.; Guo, H. The role of tumor suppressor gene SOX11 in prostate cancer. Tumor Biol. 2015, 36, 6133-6138. [CrossRef] [PubMed]

58. Pugongchai, A.; Bychkov, A.; Sampatanukul, P. Promoter hypermethylation ofSOX11correlates with adverse clinicopathological features of human prostate cancer. Int. J. Exp. Pathol. 2017, 98, 341-346. [CrossRef]

59. Sun, H.; Zhou, H.; Zhang, Y.; Chen, J.; Han, X.; Huang, D.; Ren, X.; Jia, Y.; Fan, Q.; Tian, W.; et al. Aberrant methylation of FAT4 and SOX11 in peripheral blood leukocytes and their association with gastric cancer risk. J. Cancer 2018, 9, 2275-2283. [CrossRef]

60. Kelsey, K.T.; Wiencke, J.K. Immunomethylomics: A Novel Cancer Risk Prediction Tool. Ann. Am. Thorac. Soc. 2018, 15, S76-S80. [CrossRef]

61. Levine, M.E.; Lu, A.T.; Quach, A.; Chen, B.H.; Assimes, T.L.; Bandinelli, S.; Hou, L.; Baccarelli, A.A.; Stewart, J.D.; Li, Y.; et al. An epigenetic biomarker of aging for lifespan and healthspan. Aging 2018, 10, 573-591. [CrossRef]

62. Lu, A.T.; Quach, A.; Wilson, J.G.; Reiner, A.P.; Aviv, A.; Raj, K.; Hou, L.; Baccarelli, A.A.; Li, Y.; Stewart, J.D.; et al. DNA methylation GrimAge strongly predicts lifespan and healthspan. Aging 2019, 11, 303-327. [CrossRef] [PubMed]

63. Joehanes, R.; Just, A.; Marioni, R.; Pilling, L.C.; Reynolds, L.; Mandaviya, P.R.; Guan, W.; Xu, T.; Elks, C.E.; Aslibekyan, S.; et al. Epigenetic Signatures of Cigarette Smoking. Circ. Cardiovasc. Genet. 2016, 9, 436-447. [CrossRef]

64. Park, S.L.; Patel, Y.M.; Loo, L.W.M.; Mullen, D.J.; Offringa, I.A.; Maunakea, A.; Stram, D.O.; Siegmund, K.; Murphy, S.E.; Tiirikainen, M.; et al. Association of internal smoking dose with blood DNA methylation in three racial/ethnic populations. Clin. Epigenet. 2018, 10, 1-12. [CrossRef] [PubMed]

65. Koestler, D.C.; Usset, J.L.; Christensen, B.C.; Marsit, C.J.; Karagas, M.R.; Kelsey, K.T.; Wiencke, J.K. DNA Methylation-Derived Neutrophil-to-Lymphocyte Ratio: An Epigenetic Tool to Explore Cancer Inflammation and Outcomes. Cancer Epidemiol. Biomarkers Prev. 2017, 26, 328-338. [CrossRef] [PubMed]

66. Ambatipudi, S.; Langdon, R.; Richmond, R.C.; Suderman, M.; Koestler, D.C.; Kelsey, K.T.; Kazmi, N.; Penfold, C.; Ho, K.M.; McArdle, W.; et al. DNA methylation derived systemic inflammation indices are associated with head and neck cancer development and survival. Oral Oncol. 2018, 85, 87-94. [CrossRef]

67. Grieshober, L.; Graw, S.; Barnett, M.J.; Thornquist, M.D.; Goodman, G.E.; Chen, C.; Koestler, D.C.; Marsit, C.; Doherty, J.A. Methylation-derived Neutrophil-to-Lymphocyte Ratio and Lung Cancer Risk in Heavy Smokers. Cancer Prev. Res. 2018, 11, 727-734. [CrossRef]

68. Ligthart, S.; WHI-EMPC Investigators; Marzi, C.; Aslibekyan, S.; Mendelson, M.; Conneely, K.N.; Tanaka, T.; Colicino, E.; Waite, L.L.; Joehanes, R.; et al. DNA methylation signatures of chronic low-grade inflammation are associated with complex diseases. Genome Biol. 2016, 17, 1-15. [CrossRef] 